\title{
Particle Distribution Algorithm for Solving Optimal Reactive Power Dispatch Problem
}

\author{
Lenin $\mathrm{K}^{1 *}$, Ravindhranath Reddy $\mathbf{B}^{2}$, Surya Kalavathi $\mathbf{M}^{3}$ \\ ${ }^{1}$ Researcher, JNTU, Hyderabad, India. \\ ${ }^{2}$ Deputy Executive Engineer, JNTU, Hyderabad, India. \\ ${ }^{3}$ Professor, Department of Electrical and Electronics Engineering, JNTU, Hyderabad, India.
}

Received: January 06, 2017; Accepted: January 25, 2017; Published: January 31, 2017

*Corresponding author: Lenin K, Researcher, JNTU, Hyderabad, 500 085, India, Tel: 91-9677350862; E-mail: gklenin@gmail.com

\section{Abstract}

In this paper, a novel perception of the particle swarm optimization (PSO) system called Particle Distribution Algorithm (PDA) is proposed to solve Reactive power problem. In that swarm individual particles are moved away, but discreetly characterize each particle by a field or by distribution \& its behaviour absolutely dissimilar from the family of old PSO scheme. The projected PDA has been tested in standard IEEE 30 bus test system and simulation results show clearly the better performance of the proposed algorithm in reducing the real power loss.
\end{abstract}

\section{Keywords}

Particle Swarm Optimization; Particle Distribution; Optimal Reactive Power; Transmission Loss;

\section{Introduction}

Various numerical methods like the gradient method [1, 2] Newton method [3] and linear programming [4-7] have been utilized to solve the optimal reactive power dispatch problem. The problem of voltage stability and collapse play a key role in power system planning and operation [8]. Enhancing the voltage stability, voltage magnitudes within the limits alone will not be a reliable indicator to indicate that, how far an operating point is from the collapse point. The reactive power support and voltage problems are internally related to each other.This paper formulates by combining both the real power loss minimization and maximization of Static Voltage Stability Margin (SVSM) as the objectives. Numerous Evolutionary algorithms have been already utilized to solve the reactive power flow problem [9-20]. This paper proposes Particle Distribution Algorithm (PDA) to solve reactive power dispatch problem. Proposed algorithm has better exploration and exploitation capabilities in searching the global near optimal solution \& each particle can be abstracted by a field or distribution. The parameters of the distribution are modernized by using a scheme alike to Thompson's sampling, leading to a completely new and unique perspective on particle swarm systems [21-40]. Proposed PDA has been evaluated in standard IEEE 30 bus test system. Simulation results show that our proposed approach outperforms all the entitled reported algorithms in minimization of real power loss and voltage profile index is enhanced.

\section{Voltage Stability Evaluation}

\section{Modal analysis for voltage stability evaluation}

Modal analysis is one among best methods for voltage stability enhancement in power systems. The steady state system power flow equations are given by.

$$
\left[\begin{array}{c}
\Delta P \\
\Delta Q
\end{array}\right]=\left[\begin{array}{cc}
\mathrm{J}_{\mathrm{p} \theta} & \mathrm{J}_{\mathrm{pv}} \\
\mathrm{J}_{\mathrm{q} \theta} & \mathrm{J}_{\mathrm{QV}}
\end{array}\right]\left[\begin{array}{c}
\Delta \theta \\
\Delta V
\end{array}\right]
$$

Where

$\Delta \mathrm{P}=$ Incremental change in bus real power.

$\Delta Q=$ Incremental change in bus reactive Power injection

$\Delta \theta=$ incremental change in bus voltage angle.

$\Delta \mathrm{V}=$ Incremental change in bus voltage Magnitude

Jp $\theta, J P V, J Q \theta, J Q V$ jacobian matrix are the sub-matrixes of the System voltage stability is affected by both $P$ and $Q$.

To reduce (1), let $\Delta \mathrm{P}=0$, then.

$$
\begin{aligned}
& \Delta \mathrm{Q}=\left[\mathrm{J}_{\mathrm{QV}}-\mathrm{J}_{\mathrm{Q} \theta} \mathrm{J}_{\mathrm{P} \theta^{-1}} \mathrm{~J}_{\mathrm{PV}}\right] \Delta \mathrm{V}=\mathrm{J}_{\mathrm{R}} \Delta \mathrm{V} \\
& \Delta \mathrm{V}=\mathrm{J}^{-1}-\Delta \mathrm{Q}
\end{aligned}
$$

Where

$$
\mathrm{J}_{\mathrm{R}}=\left(\mathrm{J}_{\mathrm{QV}}-\mathrm{J}_{\mathrm{Q} \theta} \mathrm{J}_{\mathrm{P} \theta^{-1}} \mathrm{JPV}\right)
$$

$\mathrm{J}_{\mathrm{R}}$ is called the reduced Jacobian matrix of the system.

\section{Modes of Voltage instability}

Voltage Stability characteristics of the system have been identified by computing the Eigen values and Eigen vectors.

Let

$\mathrm{J}_{\mathrm{R}}=\xi \Lambda \eta$

Where, 
$\xi=$ right eigenvector matrix of JR

$\eta=$ left eigenvector matrix of JR

$\Lambda=$ diagonal eigenvalue matrix of JR and

$$
\mathrm{J}_{\mathrm{R}^{-1}}=\xi \Lambda^{-1} \eta
$$

From (5) and (8), we have

$$
\begin{aligned}
& \Delta \mathrm{V}=\xi \Lambda^{-1} \eta \Delta \mathrm{Q} \\
& \Delta \mathrm{V}=\sum \frac{\text { or }_{i} \mathrm{c}_{\mathrm{i}}}{\ddot{\mathrm{e}}} \Delta \mathrm{Q}
\end{aligned}
$$

Where $\xi i$ is the ith column right eigenvector and $\eta$ the ith row left eigenvector of JR.

$\lambda \mathrm{i}$ is the ith Eigen value of JR.

The ith modal reactive power variation is,

$$
\Delta \mathrm{Q}_{\mathrm{mi}}=\mathrm{K}_{1} \xi_{\mathrm{l}}
$$

where,

$$
\mathrm{K}_{\mathrm{i}}=\sum_{\mathrm{j}} \xi_{\mathrm{ij}}-1
$$

Where

$\xi j i$ is the jth element of $\xi i$

The corresponding ith modal voltage variation is

$$
\Delta \mathrm{V}_{\mathrm{mi}}=\left[1 / \lambda_{\mathrm{i}}\right] \Delta \mathrm{Q}_{\mathrm{mi}}
$$

If $|\lambda \mathrm{i}|=0$ then the ith modal voltage will collapse.

In (10), let $\Delta \mathrm{Q}=\mathrm{ek}$ where ek has all its elements zero except the kth one being 1 . Then,

$$
\Delta \mathrm{V}=\sum_{\mathrm{i}} \frac{\eta_{1 \mathrm{k} \xi_{1}}}{\ddot{\mathrm{e}}_{1}}
$$

$\eta_{1 \mathrm{k}} \mathrm{k}$ th element of $\eta_{1}$

$\mathrm{V}-\mathrm{Q}$ sensitivity at bus $\mathrm{k}$

$$
\frac{\partial V_{K}}{\partial Q_{\mathrm{K}}}=\sum_{\mathrm{i}} \frac{\eta_{1 \mathrm{k} \xi_{1}}}{\lambda_{1}}=\sum_{\mathrm{i}} \frac{\mathrm{P}_{\mathrm{ki}}}{\lambda_{1}}
$$

\section{Problem Formulation}

The objectives of the reactive power dispatch problem is to minimize the system real power loss and maximize the Static Voltage Stability Margins (SVSM).

\section{Minimization of Real Power Loss}

Minimization of the real power loss $\left(\mathrm{P}_{\text {loss }}\right)$ in transmission lines is mathematically stated as follows.

$$
\mathrm{P}_{\text {loss }=} \sum_{\substack{k=1 \\ k=(i, j)}}^{\mathrm{n}} \mathrm{g}_{\left.\mathrm{k}_{\left(\mathrm{V}_{\hat{\mathbf{e}}}^{2}\right.}+\mathrm{V}_{\mathrm{j}}^{2}-2 \mathrm{~V}_{\mathrm{i}} \mathrm{V}_{\mathrm{j} \cos } \mathrm{ij}\right)}
$$

Where $\mathrm{n}$ is the number of transmission lines, $\mathrm{gk}$ is the conductance of branch $\mathrm{k}, \mathrm{Vi}$ and $\mathrm{Vj}$ are voltage magnitude at bus $\mathrm{i}$ and bus $\mathrm{j}$, and $\theta \mathrm{ij}$ is the voltage angle difference between bus $\mathrm{i}$ and bus $j$.

\section{Minimization of Voltage Deviation}

Minimization of the voltage deviation magnitudes (VD) at load buses is mathematically stated as follows.

$$
\text { Minimize VD }=\sum_{\mathrm{k}=1}^{\mathrm{nl}}\left|\mathrm{V}_{\mathrm{k}}-1.0\right|
$$

Where $\mathrm{nl}$ is the number of load busses and $\mathrm{Vk}$ is the voltage magnitude at bus $\mathrm{k}$.

\section{System Constraints}

Objective functions are subjected to these constraints shown below.

Load flow equality constraints:

$$
\begin{gathered}
P_{G i}-P_{D i}-V_{i} \sum_{j=1}^{n b} v_{j} \\
{\left[\begin{array}{cc}
G_{i j} & \cos \theta_{i j} \\
+B_{i j} & \sin \theta_{i j}
\end{array}\right]=0, i=1,2 \ldots, n b} \\
Q_{G i}-Q_{D i}-V_{i} \sum_{j=1}^{n b} v_{j}\left[\begin{array}{cc}
G_{i j} & \sin \theta_{i j} \\
+B_{i j} & \cos \theta_{i j}
\end{array}\right]=0, i=1,2 \ldots, n b
\end{gathered}
$$

where, nb is the number of buses, $\mathrm{PG}$ and $\mathrm{QG}$ are the real and reactive power of the generator, $\mathrm{PD}$ and $\mathrm{QD}$ are the real and reactive load of the generator, and Gij and Bij are the mutual conductance and susceptance between bus $i$ and bus $j$.

Generator bus voltage (VGi) inequality constraint:

$$
\mathrm{V}_{\mathrm{Gi}}^{\min } \leq \mathrm{V}_{\mathrm{Gi}} \leq \mathrm{V}_{\mathrm{Gi}}^{\mathrm{max}}, \mathrm{i} \in \mathrm{ng}
$$

Load bus voltage (VLi) inequality constraint:

$$
\mathrm{V}_{\mathrm{Li}}^{\min } \leq \mathrm{V}_{\mathrm{Li}} \leq \mathrm{V}_{\mathrm{Li}}^{\max }, \mathrm{i} \in \mathrm{nl}
$$

Switchable reactive power compensations (QCi) inequality constraint:

$$
\mathrm{Q}_{\mathrm{Ci}}^{\min } \leq \mathrm{Q}_{\mathrm{Ci}} \leq \mathrm{Q}_{\mathrm{Ci}}^{\max }, \mathrm{i} \in \mathrm{nc}
$$

Reactive power generation (QGi) inequality constraint:

$$
\mathrm{Q}_{\mathrm{Gi}}^{\min } \leq \mathrm{Q}_{\mathrm{Gi}} \leq \mathrm{Q}_{\mathrm{Gi}}^{\max }, \mathrm{i} \in \mathrm{ng}
$$

Transformers tap setting (Ti) inequality constraint:

$$
\mathrm{T}_{\mathrm{i}}^{\min } \leq \mathrm{T}_{\mathrm{i}} \leq \mathrm{T}_{\mathrm{i}}^{\max }, \mathrm{i} \in \mathrm{nt}
$$

Transmission line flow (SLi) inequality constraint:

$$
\mathrm{S}_{\mathrm{Li}}^{\min } \leq \mathrm{S}_{\mathrm{Li}}^{\max }, \mathrm{i} \in \mathrm{nl}
$$

Where, nc, ng and nt are numbers of the switchable reactive power sources, generators and transformers 


\section{Particle Distribution Algorithm (PDA)}

In the basic particle swarm optimization (PSO) model, an individual particle consists of: A current position, a current velocity and a personal best found position. Each of these components is required in order to decide the succeeding position of the particle for the subsequent iteration. The particle's position in the succeeding iteration depends upon its present position and the particle's velocity. The modernized velocity, in turn, depends on the particle's current velocity, the particle's personal best found position and the population's global best found position. The representation of a swarm of particles "flying" through space no longer aptly describes the high-level concept of the algorithm. Rather, the algorithm now consists of a population of "particle fields" which move throughout the space in a different way. Because the "positions" of these "particle fields" are defined as arbitrary distributions, "evaluating" a "particle field's" existing "position" is nondeterministic, and so these "particle fields" do not certainly have to "move" to discover new points. These "particle fields" remain "stationary" in the space until either the individual's personal best point changes, or the population's global best point changes. This population of "particle fields" can, itself, be alleged as an arbitrary field of particles defined as a mixture distribution made up of each individual distribution. This population level distribution can be thought of as an abstract depiction of a particle swarm, representing a probability distribution of all possible particle locations for the consequent iteration. With this new outlook, it is possible to discover new directions in refining or altering the behaviour of the algorithm. With these changes taken into account, we have now moved away from the traditional PSO paradigm and arrived at a new, distinct algorithm, which will be hereafter referred to as Particle Distribution Algorithm (PDA). This algorithm consists of a population of "particle field" individuals and a "point pool" of candidate solution points. The population of particle field individuals uses PSO principles to guide the exploration of the solution space, which is carried out by creating and weighing, the pool of candidate solution points. Similar to traditional PSO algorithms, the PDA algorithm consists of an initialization phase and a simulation phase which loops until some end criteria is met, at which point the best solution found by the algorithm is returned as output. A particle field individual is designated at an arbitrary position from the population, according to some weighting scheme. Then, the point is produced by sampling the arbitrary distribution defined by the selected individual. This arbitrary distribution is constructed using the individual's personal best, and the global best points.

Given a particle field with personal best point $\overrightarrow{\mathrm{p}}_{\mathrm{i}}$ and for which the global best point is $\vec{p}_{g}$, the position of the candidate solution point, $\overrightarrow{\mathrm{c}}$ is determined according to:

$$
\begin{aligned}
& \overrightarrow{\mathrm{p}}_{\mathrm{m}}=\frac{\overrightarrow{\mathrm{p}}_{\mathrm{i}}+\overrightarrow{\mathrm{p}}_{\mathrm{g}}}{2} \\
& \overrightarrow{\sigma^{2}}=\left|\overrightarrow{p_{i}}-\overrightarrow{p_{g}}\right|
\end{aligned}
$$

$$
\overrightarrow{\mathrm{c}}=\overrightarrow{\mathrm{N}}\left(\overrightarrow{\mathrm{p}_{\mathrm{m}}}-\overrightarrow{\sigma^{2}}\right)
$$

Once the candidate solution has been produced, the objective function is weighed, using this produced point as its input, in order to assign it a value. After each candidate solution in the point pool has been produced, the second phase begins. In this phase, the population of particle field individuals is modernized. Each individual updates its own best found point using the set of candidate solutions produced from its own distribution. Each individual selects the best point from the set of associated candidate solutions. If the best associated candidate solution is better than the individual's personal best found point, the individual sets its personal best found point to be equal to that candidate solution point. The pool of candidate solutions is then "emptied", and the simulation endures to the succeeding iteration. Once the termination criteria have been met, the global best found point is returned as output of the algorithm.

\section{Input:}

Function $\mathrm{f}(\mathrm{)}$ to be optimized

Initialization range lbound, ubound

Particle field population size pop

Candidate solution point pool size $\mathrm{n}_{\text {pool }}$

Weighting function ù ()

\section{Output:}

Point $\overrightarrow{\mathrm{p}}_{\mathrm{i}}$ representing best found solution

\section{Method:}

Generate particle field population $\mathrm{P}$ with size $\mathrm{n}_{\text {pop }}$ Generate candidate solution point pool C with size $n_{\text {pool }}$ For each particle field $i \in p$ do

$\overrightarrow{\mathrm{p}}_{\mathrm{i}}=\overrightarrow{\mathrm{U}}$ [lbound, ubound]

If $\mathrm{f}\left(\overrightarrow{\mathrm{p}}_{\mathrm{i}}\right)<f\left(\overrightarrow{\mathrm{p}}_{\mathrm{j}}\right)$ Then

$\overrightarrow{\mathrm{p}}_{\mathrm{i}} \Leftarrow \overrightarrow{\mathrm{p}}_{\mathrm{j}}$

End if

End for

While termination criteria not met do

For each candidate solution point $\overrightarrow{\mathrm{c}} \in \mathrm{C}$ do

Select particle field $i \in \mathrm{p}_{\text {with probability }} \frac{\grave{\mathrm{u}}(\mathrm{i})}{\sum \mathrm{p} \in \mathrm{P}^{\dot{u}(\mathrm{p})}}$

$\overrightarrow{\mathrm{c}} \Leftarrow \overrightarrow{\mathrm{N}}\left(\frac{\overrightarrow{\mathrm{p}_{\mathrm{i}}}+\overrightarrow{\mathrm{p}_{\mathrm{g}}}}{2},\left[\overrightarrow{\mathrm{p}_{\mathrm{i}}}-\overrightarrow{\mathrm{p}_{\mathrm{g}}}\right]\right)$

$\overrightarrow{\mathrm{s}}_{\mathrm{i}} \Leftarrow \mathrm{s}_{\mathrm{i}} \cup\{\overrightarrow{\mathrm{c}}\}$ 


\section{End for}

For each particle field $\mathrm{i} \in \mathrm{p}$ do

Choose point $\overrightarrow{\mathrm{c}}_{\text {min }}$ from $\mathrm{S}_{\mathrm{i}}$ which minimizes $\mathrm{f}\left(\overrightarrow{\mathrm{c}}_{\text {min }}\right)$

If $\mathrm{f}\left(\overrightarrow{\mathrm{c}}_{\min }<f(\mathrm{p})_{\mathrm{i}}\right)$ then

$\overrightarrow{\mathrm{p}}_{\mathrm{i}} \Leftarrow \overrightarrow{\mathrm{c}}_{\min }$

If $\mathrm{f}\left(\overrightarrow{\mathrm{p}}_{\mathrm{i}}\right)<f\left(\overrightarrow{\mathrm{p}}_{\mathrm{j}}\right)$

Then $\overrightarrow{\mathrm{p}}_{\mathrm{i}} \Leftarrow \overrightarrow{\mathrm{p}}_{\mathrm{j}}$

End if

End if

$\mathrm{S}_{\mathrm{i}} \Leftarrow \varnothing$

End for

End

\section{Simulation Results}

The efficiency of the proposed Particle Distribution Algorithm (PDA) is demonstrated by testing it on standard IEEE-30 bus system. The IEEE-30 bus system has 6 generator buses, 24 load buses and 41 transmission lines of which four branches are (6-9), (6-10) , (4-12) and (28-27) - are with the tap setting transformers. The lower voltage magnitude limits at all buses are 0.95 p.u. and the upper limits are 1.1 for all the PV buses and 1.05 p.u. for all the $\mathrm{PQ}$ buses and the reference bus. The simulation results have been presented in Tables 1, 2, $3 \& 4$. And in the Table 5 shows the proposed algorithm powerfully reduces the real power losses when compared to other given algorithms. The optimal values of the control variables along with the minimum loss obtained are given in Table 1. Corresponding to this control variable setting, it was found that there are no limit violations in any of the state variables.

Table 1: Results of PDA - ORPD optimal control variables

\begin{tabular}{|c|c|}
\hline Control Variables & Variable Setting \\
\hline V1 & 1.047 \\
V2 & 1.046 \\
V5 & 1.041 \\
V8 & 1.030 \\
V11 & 1.004 \\
V13 & 1.031 \\
T11 & 1.00 \\
T12 & 1.00 \\
T15 & 1.01 \\
T36 & 1.01 \\
Qc10 & 3 \\
Qc12 & 3 \\
Qc15 & 2 \\
Qc17 & 0 \\
Qc20 & 2 \\
Qc23 & 3 \\
Qc24 & 3 \\
Qc29 & 2 \\
Real power loss & 4.2869 \\
SVSM & 0.2479 \\
\hline
\end{tabular}

\begin{tabular}{|c|c|}
$\begin{array}{c}\text { Table 2: Results of PDA -Voltage Stability Control Reactive Power Dispatch } \\
\text { Optimal Control Variables }\end{array}$ \\
\hline Control Variables & Variable Setting \\
\hline V1 & 1.049 \\
V2 & 1.047 \\
V5 & 1.044 \\
V8 & 1.032 \\
V11 & 1.005 \\
V13 & 1.034 \\
T11 & 0.090 \\
T12 & 0.090 \\
T15 & 0.090 \\
T36 & 0.090 \\
Qc10 & 3 \\
Qc12 & 3 \\
Qc15 & 2 \\
Qc17 & 3 \\
Qc20 & 0 \\
Qc23 & 2 \\
Qc24 & 2 \\
Qc29 & 3 \\
Real power loss & 4.9896 \\
SVSM & 0.2484 \\
\hline
\end{tabular}

Table 3: Voltage Stability under Contingency State

\begin{tabular}{|c|c|c|c|}
\hline Sl.No & Contingency & ORPD Setting & VSCRPD Setting \\
\hline 1 & $28-27$ & 0.1419 & 0.1434 \\
\hline 2 & $4-12$ & 0.1642 & 0.1650 \\
\hline 3 & $1-3$ & 0.1761 & 0.1772 \\
\hline 4 & $2-4$ & 0.2022 & 0.2043 \\
\hline
\end{tabular}

Optimal Reactive Power Dispatch problem together with voltage stability constraint problem was handled in this case as a multi-objective optimization problem where both power loss and maximum voltage stability margin of the system were optimized simultaneously. Table 2 indicates the optimal values of these control variables. Also it is found that there are no limit violations of the state variables. It indicates the voltage stability index has increased from 0.2479 to 0.2484 , an advance in the system voltage stability. To determine the voltage security of the system, contingency analysis was conducted using the control variable setting obtained in case 1 and case 2. The Eigen values equivalents to the four critical contingencies are given in Table 3. From this result it is observed that the Eigen value has been improved considerably for all contingencies in the second case.

\section{Conclusion}

In this paper, Particle Distribution Algorithm (PDA) has been successfully applied to solve Optimal Reactive Power Dispatch problem. The proposed algorithm has been tested on the standard IEEE 30 bus system. Simulation results show the heftiness of projected Particle Distribution Algorithm (PDA) method in decreasing the real power loss \& voltage profile index has been enhanced. The control variables obtained after the optimization by Particle Distribution Algorithm (PDA) is within the limits. 
Table 4: Limit Violation Checking Of State Variables

\begin{tabular}{|c|c|c|c|c|}
\hline \multirow{2}{*}{ State variables } & \multicolumn{2}{|c|}{ limits } & \multirow{2}{*}{ ORPD } & \multirow{2}{*}{ VSCRPD } \\
\cline { 1 - 3 } Q1 & Lower & upper & & \\
\hline Q2 & -20 & 152 & 1.3422 & -1.3269 \\
\hline Q5 & -20 & 61 & 8.9900 & 9.8232 \\
\hline Q8 & -15 & 49.92 & 25.920 & 26.001 \\
\hline Q11 & -10 & 63.52 & 38.8200 & 40.802 \\
\hline Q13 & -15 & 42 & 2.9300 & 5.002 \\
\hline V3 & -15 & 48 & 8.1025 & 6.033 \\
\hline V4 & 0.95 & 1.05 & 1.0372 & 1.0392 \\
\hline V6 & 0.95 & 1.05 & 1.0307 & 1.0328 \\
\hline V7 & 0.95 & 1.05 & 1.0282 & 1.0298 \\
\hline V9 & 0.95 & 1.05 & 1.0101 & 1.0152 \\
\hline V10 & 0.95 & 1.05 & 1.0462 & 1.0412 \\
\hline V12 & 0.95 & 1.05 & 1.0482 & 1.0498 \\
\hline V14 & 0.95 & 1.05 & 1.0400 & 1.0466 \\
\hline V15 & 0.95 & 1.05 & 1.0474 & 1.0443 \\
\hline V16 & 0.95 & 1.05 & 1.0457 & 1.0413 \\
\hline V17 & 0.95 & 1.05 & 1.0426 & 1.0405 \\
\hline V18 & 0.95 & 1.05 & 1.0382 & 1.0396 \\
\hline V19 & 0.95 & 1.05 & 1.0392 & 1.0400 \\
\hline V20 & 0.95 & 1.05 & 1.0381 & 1.0394 \\
\hline V21 & 0.95 & 1.05 & 1.0112 & 1.0194 \\
\hline V22 & 0.95 & 1.05 & 1.0435 & 1.0243 \\
\hline V23 & 0.95 & 1.05 & 1.0448 & 1.0396 \\
\hline V24 & 0.95 & 1.05 & 1.0472 & 1.0372 \\
\hline V25 & 0.95 & 1.05 & 1.0484 & 1.0372 \\
\hline V26 & 0.95 & 1.05 & 1.0142 & 1.0192 \\
\hline V27 & 0.95 & 1.05 & 1.0494 & 1.0422 \\
\hline V28 & 0.95 & 1.05 & 1.0472 & 1.0452 \\
\hline V29 & 0.95 & 1.05 & 1.0243 & 1.0283 \\
\hline V30 & 0.95 & 1.05 & 1.0439 & 1.0419 \\
\hline & & & 1.0418 & 1.0397 \\
\hline
\end{tabular}

Table 5: Comparison of Real Power Loss

\begin{tabular}{|c|c|}
\hline Method & Minimum loss \\
\hline Evolutionary programming [41] & 5.0159 \\
\hline Genetic algorithm [42] & 4.665 \\
\hline Real coded GA with Lindex as SVSM [43] & 4.568 \\
\hline Real coded genetic algorithm [44] & 4.5015 \\
\hline Proposed PDA method & 4.2869 \\
\hline
\end{tabular}

\section{References}

1. Alsac 0 and Scott B. Optimal load flow with steady state security. IEEE Transaction. 1973;93(3):745-751. DOI: 10.1109/TPAS.1974.293972.

2. Lee KY, Paru YM, Oritz JL. A united approach to optimal real and reactive power dispatch. IEEE Transactions on Power Apparatus and Systems. 1985;104(5):1147-1153. DOI: 10.1109/TPAS.1985.323466.

3. Monticelli A, Pereira MVF. Granville S. Security constrained optimal power flow with post contingency corrective rescheduling. IEEE Transactions on Power Systems. 1987;2(1):175-182. DOI: 10.1109/ TPWRS.1987.4335095
4. Deeb N, Shahidehpur SM. Linear reactive power optimization in a large power network using the decomposition approach. IEEE Transactions on Power Systems. 1990;5(2):428-435.

5. Hobson E. Network consrained reactive power control using linear programming. IEEE Transactions on Power Systems. 1980;99(3):868877. DOI: 10.1109/TPAS.1980.319715.

6. Lee KY, Park YM, Oritz JL. Fuel -cost optimization for both real and reactive power dispatches. IEE Proc. 1984;131(3):85-93. DOI: 10.1049/ip-c:19840012.

7. Mangoli MK, Lee KY. Optimal real and reactive power control using linear programming. Electr Power Syst Res. 1993;26(1):1-10.

8. Canizares CA, De Souza ACZ, Quintana VH. Comparison of performance indices for detection of proximity to voltage collapse. IEEE Transactions on Power Systems. 1996;11(3):1441-1450. DOI: $10.1109 / 59.535685$.

9. Berizzi A, Bovo C, Merlo M, Delfanti M. A GA approach to compare ORPF objective functions including secondary voltage regulation. Electric Power Systems Research. 2012;84(1):187-194.

10. Devaraj D, Yeganarayana B. Genetic algorithm based optimal power flow for security enhancement. IEE Proceedings-Generation, Transmission and Distribution. 2005;152(6):899-905. DOI: 10.1049/ ip-gtd:20045234.

11. Berizzi A, Bovo C, Merlo M, Delfanti M. A GA approach to compare ORPF objective functions including secondary voltage regulation. Electric Power Systems Research. 2012;84(1):187-194.

12. Yang CF, Lai GG, Lee $\mathrm{CH}$, Su CT, Chang GW. Optimal setting of reactive compensation devices with an improved voltage stability index for voltage stability enhancement. International Journal of Electrical Power and Energy Systems. 2012;37(1):50-57.

13. Roy P, Ghoshal S, Thakur S. Optimal var control for improvements in voltage profiles and for real power loss minimization using biogeography based optimization. International Journal of Electrical Power and Energy Systems. 2012;43(1):830-838.

14. Venkatesh B, Sadasivam G, Khan M. A new optimal reactive power scheduling method for loss minimization and voltage stability margin maximization using successive multi-objective fuzzy lp technique. IEEE Transactions on Power Systems. 2000;15(2):844-851. DOI: $10.1109 / 59.867183$

15. Yan W, Lu S, Yu D. A novel optimal reactive power dispatch method based on an improved hybrid evolutionary programming technique. IEEE Transactions on Power Systems. 2004;19(2):913-918. DOI: 10.1109/TPWRS.2004.826716

16. Yan W, Liu F, Chung C, Wong K. A hybrid genetic algorithminterior point method for optimal reactive power flow. IEEE Transactions on Power Systems. 2006;21(3):1163-1169. DOI: 10.1109/ TPWRS.2006.879262.

17. Yu J, Yan W, Li W, Chung C, Wong K. An unfixed piecewiseoptimal reactive power-flow model and its algorithm for ac-dc systems. IEEE Transactions on Power Systems. 2008;23(1):170-176. DOI: 10.1109/ TPWRS.2007.907387.

18. Capitanescu F. Assessing reactive power reserves with respect to operating constraints and voltage stability. IEEE Transactions on Power Systems. 2011;26(4):2224-2234. DOI: 10.1109/ TPWRS.2011.2109741.

19. Hu Z, Wang X, Taylor G. Stochastic optimal reactive power dispatch: Formulation and solution method. International Journal of Electrical Power and Energy Systems. 2010;32(6):615-621. 
20. Kargarian A, Raoofat M, Mohammadi M. Probabilistic reactive power procurement in hybrid electricity markets with uncertain loads. Electric Power Systems Research. 2012;82(1):68-80.

21. Angeline PJ. Using selection to improve particle swarm optimization presented at the IEEE International Conference on Computational Intelligence. 1998. DOI: 10.1109/ICEC.1998.699327.

22. Clerc M, Kennedy J. The particle swarm - explosion, stability, and convergence in a multidimensional complex space. IEEE Transactions on Evolutionary Computation. 2002;6(1):58-73. DOI: $10.1109 / 4235.985692$.

23. Bell N. Swarm optimization using agents modeled as distributions MCS Thesis, Carelton University. 2014.

24.Eberhart R, Shi Y. Comparing inertia weights and constriction factors in particle swarm optimization. Congress on Evolutionary Computation. 2000;1:84-88.

25. Higashi N, Iba H. Particle swarm optimization with Gaussian mutation. presented at the IEEE Swarm Intelligence Symposium, Indianapolis. 2003. DOI: $10.1109 /$ SIS.2003.1202250.

26. Kennedy J. Bare bones particle swarms. presented at the IEEE Swarm Intelligence Symposium. 2003. DOI: 10.1109/SIS.2003.1202251.

27. Kennedy J, Eberhart R. Particle swarm optimization. presented at the IEEE International Conference on Neural Networks. 1995. DOI: 10.1109/ICNN.1995.488968

28. Kennedy J, Mendes R. Population structure and particle swarm performance. Congress on Evolutionary Computation. 2002;2:16711676. DOI: 10.1109/CEC.2002.1004493.

29. Locatelli M. A note on the griewank test function. Journal of Global Optimization. 2003;25(2):169-174. DOI: 10.1023/A:1021956306041.

30. Lovbjerg M, Rasmussen TK, Krink T. Hybrid particle swarm optimiser with breeding and subpopulations. presented at the Genetic and Evolutionary Computation Conference. 2001.

31. Mendes R, Kennedy J, Neves J. The fully informed particle swarm: Simpler, maybe better. IEEE Transactions on Evolutionary Computation. 2004;8(3):204-210. DOI: 10.1109/TEVC.2004.826074.

32. Monson CK, Seppi KD. Exposing origin-seeking bias in pso. presented at the Conference on Genetic and Evolutionary Computation, GECCO '05. 2005:241-248. Doi:10.1145/1068009.1068045.
33. Ozcan E, Mohan CK. Analysis of a simple particle swarm optimization system. Intelligent Engineering Systems Through Artificial Neural Networks. 1988;8:253-258.

34. Ozcan E, Mohan CK. Particle swarm optimization: Surfing the waves. Congress on Evolutionary Computation. 1999;3:1939-1944. DOI: 10.1109/CEC.1999.785510.

35. Ratnaweera A, Halgamuge S, Watson HC. Self-organizing hierarchical particle swarm optimizer with time-varying acceleration coefficients. IEEE Transactions on Evolutionary Computation. 2004;8(3):240-255.

36. Settles M, Soule T. Breeding swarms: A ga/pso hybrid. presented at the Conference on Genetic and Evolutionary Computation, GECCO ' 05 . 2005:161-168. Doi:10.1145/1068009.1068035.

37. Shi Y, Eberhart R. Empirical study of particle swarm optimization. Congress on Evolutionary Computation. 1999;3:1945-1950. DOI: 10.1109/CEC.1999.785511.

38. Spears WM, Green DT, Spears DF. Biases in particle swarm optimization. Int J Swarm Intell Res. 2010;1(2):34-57. DOI: 10.4018/978-1-46661592-2.ch002.

39. Zhan ZH, Zhang J, Chung HSH. Adaptive particle swarm optimization. IEEE Transactions on Systems, Man, and Cybernetics, Part B: Cybernetics, IEEE Swarm Intelligence Symposium, 2003. 2009;39(6):1362-1381. DOI: 10.1109/TSMCB.2009.2015956.

40. Nathan B, John Oommen B. Particle field optimization: A new paradigm for swarm intelligence. in Proceedings of the 14th International Conference on Autonomous Agents and Multiagent Systems. 2015:257-265.

41. Wu QH, Ma JT. Power system optimal reactive power dispatch using evolutionary programming. IEEE Transactions on power systems. 1995;10(3):1243-1248. DOI: 10.1109/59.466531.

42. Durairaj S, Devaraj D, Kannan PS. Genetic algorithm applications to optimal reactive power dispatch with voltage stability enhancement. IE(I) Journal-EL. 2006;87.

43. Devaraj D. Improved genetic algorithm for multi - objective reactive power dispatch problem. European Transactions on electrical power. 2007;17:569-581.

44. Aruna Jeyanthy P, Devaraj D. Optimal Reactive Power Dispatch for Voltage Stability Enhancement Using Real Coded Genetic Algorithm. International Journal of Computer and Electrical Engineering, 2010;2(4):1793-8163. 